\title{
DARK MATTER CONTENT IN GLOBULAR CLUSTER NGC 6397
}

\author{
Jihye Shin ${ }^{1}$, Sungsoo S. Kim ${ }^{1,2}$, ANd Young-WoOK LeE ${ }^{3}$ \\ ${ }^{1}$ Department of Astronomy and Space Science, Kyung Hee University, Yongin, Kyunggi 446-701, Korea \\ E-mail : jhshin.jhshin@gmail.com \\ 2 School of Space Research, Kyung Hee University, Yongin, Kyunggi 446-701, Korea \\ 3 Center for Galaxy Evolution Research and Department of Astronomy, Yonsei University, Seoul 120-749, Korea \\ (Received June 20, 2013; Accepted July 29, 2013)
}

\begin{abstract}
We trace the dynamical evolution of dark matter (DM) content in NGC 6397, one of the native Galactic globular clusters (GCs). The relatively strong tidal field (Galactocentric radius of $\sim 6 \mathrm{kpc}$ ) and short relaxation timescale $(\sim 0.3 \mathrm{Gyr})$ of the cluster can cause a significant amount of DM particles to evaporate from the cluster in the Hubble time. Thus, the cluster can initially contain a non-negligible amount of DM. Using the most advanced Fokker-Planck (FP) method, we calculate the dynamical evolution of GCs for numerous initial conditions to determine the maximum initial DM content in NGC 6397 that matches the present-day brightness and velocity dispersion profiles of the cluster. We find that the maximum allowed initial DM mass is slightly less than the initial stellar mass in the cluster. Our findings imply that NGC 6397 did not initially contain a significant amount of DM, and is similar to that of NGC 2419, the remotest and the most massive Galactic GC.
\end{abstract}

Key words : Galaxy — evolution: Galaxy — formation: Galaxy — kinematics and dynamics: globular clusters — general: methods — numerical

\section{INTRODUCTION}

Since Peebles \& Dicke (1968) first proposed a primordial scenario for globular cluster (GC) formation, implying that GCs may have formed in individual dark matter (DM) halos right after the recombination of the universe, the picture of GC formation in the mini DM halo has been considered in many studies (Peebles 1984; Cen 2001; Beasly et al. 2003; Bekki et al. 2007; Boley et al. 2009; Griffen et al. 2011). Meanwhile, three more scenarios for GC formation have been suggested: (a) gas compression by strong shocks (Gunn 1980), (b) thermal instabilities of the hot gaseous halo (Fall \& Rees 1985), and (c) gas-rich galaxy mergers (Ashman \& Zepf 1992). In these gas-dynamical views, a diminished DM content is hypothesized as being associated with the formation of GCs, compared to that of the primordial picture.

Moore (1996) demonstrated that tidal tails around GCs cannot be formed if the GCs reside in the individual DM halos; however, some of the Galactic GCs do show tidal tails, implying that at least some of the Galactic GCs do not currently have significant DM around them. Numerical studies have also shown that even if a GC initially has a DM halo, a significant amount of DM could be tidally stripped away when GCs were being accreted to the Galaxy (Bromm \& Clarke 2002; Mashchenko \& Sills 2005; Saitoh et al. 2006). Even if the DM halo of a GC survives through

Corresponding Author: S. S. Kim the tidal stripping, the DM content associated with the GC would be gradually depleted, since the DM would migrate to the outer part of the GC due to a mass segregation with stars (Baumgardt \& Mieske 2008).

NGC 2419 is the remotest known Galactic GC from the Galactic center $(\sim 90 \mathrm{kpc})$; therefore, it should be the least influenced by Galactic tides. NGC 2419 also has the longest DM depletion timescale $t_{\mathrm{dep}}$ (further clarification in Section 2) among the Galactic GCs, which is the timescale on which the DM becomes depleted from the central region of a star cluster, due to dynamical friction and mass segregation (Baumgardt \& Mieske 2008; Baumgardt et al. 2009). For these reasons, NGC 2419 has been regarded as the best target, not only to study the existence of the present-day DM, but also to infer the amount of the initial DM content. Baumgardt et al. (2009) and Conroy, Loeb, \& Spergel (2011) reported that the maximum possible present-day total DM mass, $M_{\mathrm{DM}}$, that satisfies the observed velocity and brightness profiles of NGC 2419 is not larger than the present-day total stellar mass $M_{\text {star }}$. This result implies that only a relatively insignificant amount of DM can be associated with the initial state of NGC 2419.

It is too early to generalize the results of NGC 2419 for the entire Galactic GC population, and similar studies on other GCs would be useful in understanding the DM content during the early phases of the GCs. For this project, we studied the dynamical evolution of NGC 6397 to determine if its current brightness and ve- 
locity profiles could verify any constraints to the initial DM content in the GC. NGC 6397 is a typical Galactic GC in terms of its current mass and size. It has a relaxation timescale $t_{\mathrm{rh}}$ (see definition in Section 2) shorter than the Hubble time $(<0.3 \mathrm{Gyr})$, and orbits the Galaxy with a Galactocentric radius $R_{G}$ of $\sim 6 \mathrm{kpc}$. Unlike in NGC 2419, the DM of NGC 6397 would undergo non-negligible mass segregation due to its short $t_{\mathrm{rh}}$, thus one needs to follow the dynamical evolution of NGC 6397 to trace the evolution of the DM content in the cluster.

In this study, we performed a set of anisotropic Fokker-Planck (FP) calculations to follow the evolution of DM content in NGC 6397. We first analyzed the general evolutionary characteristics of the DM components in a GC, and then looked for the GC initial conditions that would best fit the present-day brightness and velocity profiles of NGC 6397 observed by Meylan \& Mayor (1991) and Trager et al. (1995).

This paper is organized as follows. In Section 2 we describe the improvement of our FP models for DM components. In Section 3 we analyze the behavior of the dynamical evolution of GCs with a DM component. We then determine the best-fit initial conditions and the DM content for NGC 6397, described in Section 4, and summarize our results in Section 5 .

\section{MODELS AND INITIAL CONDITIONS}

We adopted the most advanced anisotropic FP model, used in Shin, Kim, \& Takahashi (2008) and Shin et al. (2013), which was originally developed by Takahashi \& Lee (2000, and references therein). The model integrates the orbit-averaged FP equation of two (energy-angular momentum) dimensions and considers multiple stellar mass components, three-body and tidal-capture binary heating, stellar evolution, tidal fields, disk/bulge shocks, dynamical friction, and realistic (eccentric) cluster orbit. The model implements an Alternating Direction Implicit (ADI) method, developed by Shin \& Kim (2007), for integrating the twodimensional FP equation with better numerical stability.

To calculate the dynamical evolution of a $\mathrm{GC}$ with a DM component, we implemented the formulation of Takahashi et al. (2002) into the FP model. The orbitaveraged FP equation with multiple mass components can be written as follows:

$$
\begin{aligned}
A \frac{\partial g_{i}}{\partial t}= & \frac{\partial}{\partial E}\left(D_{E_{i}} g_{i}+D_{E E_{i}} \frac{\partial g_{i}}{\partial E}+D_{E R_{i}} \frac{\partial g_{i}}{\partial R}\right) \\
& +\frac{\partial}{\partial R}\left(D_{R_{i}} g_{i}+D_{R E_{i}} \frac{\partial g_{i}}{\partial E}+D_{R R_{i}} \frac{\partial g_{i}}{\partial R}\right),
\end{aligned}
$$

where $A$ is the weight function, $g_{i}$ is the mass density in phase space for mass component $i$, and $D_{E_{i}}$, $D_{R_{i}}, D_{E E_{i}}, D_{R R_{i}}, D_{E R_{i}}$, and $D_{R E_{i}}$ are the flux coefficients, which are described in detail in Takahashi (1997). These variables and coefficients are functions of energy per unit mass $E$ and scaled angular momentum
Table 1.

\begin{tabular}{|c|c|c|c|c|c|}
\hline \multirow[b]{2}{*}{ Model } & \multicolumn{4}{|c|}{ star } & \multirow{2}{*}{$\frac{\mathrm{DM}}{M_{\mathrm{DM}} / M_{\text {star }}}$} \\
\hline & $\begin{array}{l}M_{\text {star }} \\
\left(\mathrm{M}_{\odot}\right)\end{array}$ & $\begin{array}{c}r_{\mathrm{h}, \mathrm{star}} \\
(\mathrm{pc})\end{array}$ & $\begin{array}{c}t_{\mathrm{rh}, \mathrm{star}} \\
(\mathrm{Gyr}) \\
\end{array}$ & $\overline{W_{0}}$ & \\
\hline A00 & $10^{5}$ & 1.0 & 0.1 & 4 & 0.0 \\
\hline A03 & $10^{5}$ & 1.0 & 0.1 & 4 & 0.3 \\
\hline A 05 & $10^{5}$ & 1.0 & 0.1 & 4 & 0.5 \\
\hline A 10 & $10^{5}$ & 1.0 & 0.1 & 4 & 1.0 \\
\hline B05 & $10^{6}$ & 7.0 & 5.0 & 4 & 0.5 \\
\hline
\end{tabular}

FP models with different $M_{\mathrm{DM}} / M_{\text {star }}$ and $t_{\mathrm{rh} \text {,star. }}$

$R$. Since the mass of an individual DM particle $m_{\mathrm{DM}}$ is much smaller than that of a star $m_{\text {star }}$, we used a limit of $m_{\mathrm{DM}} \rightarrow 0$. Therefore, $D_{E_{\mathrm{DM}}}$ and $D_{R_{\mathrm{DM}}}$, which are proportional to $m_{\mathrm{DM}}$, approached zero, while the other coefficients, $D_{E E_{\mathrm{DM}}}, D_{R R_{\mathrm{DM}}}, D_{E R_{\mathrm{DM}}}$, and $D_{R E_{\mathrm{DM}}}$ did not vanish. Since $D_{E E}, D_{R R}, D_{E R}$, and $D_{R E}$ are independent of mass, these coefficients are the same for both the star and the DM, and we omitted the subscripts for the components of these coefficients. Thus, the FP equations read:

$$
\begin{aligned}
A \frac{\partial g_{\mathrm{star}}}{\partial t}= & \frac{\partial}{\partial E}\left(D_{E_{\mathrm{star}}} g_{\mathrm{star}}+D_{E E} \frac{\partial g_{\mathrm{star}}}{\partial E}+D_{E R} \frac{\partial g_{\mathrm{star}}}{\partial R}\right) \\
& +\frac{\partial}{\partial R}\left(D_{R_{\mathrm{star}}} g_{\mathrm{star}}+D_{R E} \frac{\partial g_{\mathrm{star}}}{\partial E}+D_{R R} \frac{\partial g_{\mathrm{star}}}{\partial R}\right) \\
A \frac{\partial g_{\mathrm{DM}}}{\partial t}= & \frac{\partial}{\partial E}\left(D_{E E} \frac{\partial g_{\mathrm{DM}}}{\partial E}+D_{E R} \frac{\partial g_{\mathrm{DM}}}{\partial R}\right) \\
& +\frac{\partial}{\partial R}\left(D_{R E} \frac{\partial g_{\mathrm{DM}}}{\partial E}+D_{R R} \frac{\partial g_{\mathrm{DM}}}{\partial R}\right)
\end{aligned}
$$

By separating the FP equation for the DM from that for the stars, as in equation (2), we were able to integrate the FP equations without encountering numerical instabilities. Although the relaxation between DM particles is negligible as $D_{E_{\mathrm{DM}}}$ and $D_{R_{\mathrm{DM}}}$ approach zero, the DM component still evolves through its interaction with the stellar component. The DM component gradually migrates to the outer part of the GC by dynamical friction and mass segregation: thus, the DM gradually becomes depleted in the inner region of the potential (where the stars reside) as the GC evolves. Baumgardt $\&$ Mieske (2008) derived $t_{\text {dep }}$ to be:

$t_{\mathrm{dep}}=5.86\left(\frac{M_{\mathrm{t}}}{10^{6} \mathrm{M}_{\odot}}\right)^{1 / 2}\left(\frac{r_{\mathrm{h}, \mathrm{star}}}{5 \mathrm{pc}}\right)^{3 / 2}\left(\frac{\bar{m}_{\mathrm{star}}}{\mathrm{M}_{\odot}}\right)^{-1} \mathrm{Gyr}$,

where $M_{\mathrm{t}}$ is the cluster's total mass $\left(M_{\mathrm{t}}=M_{\mathrm{star}}+\right.$ $\left.M_{\mathrm{DM}}\right), r_{\mathrm{h} \text {,star }}$ is the half-mass radius $r_{\mathrm{h}}$ of the stellar component, and $\bar{m}_{\text {star }}$ is the mean stellar mass. Note that $t_{\text {dep }}$ has the same dependence on $M, r_{\mathrm{h}}$ and $\bar{m}$ as the relaxation timescale at a half-mass radius of a cluster composed of only stars,

$$
t_{\mathrm{rh}, \mathrm{star}}=0.138 \frac{M_{\mathrm{star}}^{1 / 2} r_{\mathrm{h}, \mathrm{star}}^{3 / 2}}{\bar{m}_{\mathrm{star}} G^{1 / 2} \ln \Lambda}
$$

(Spitzer 1987) 

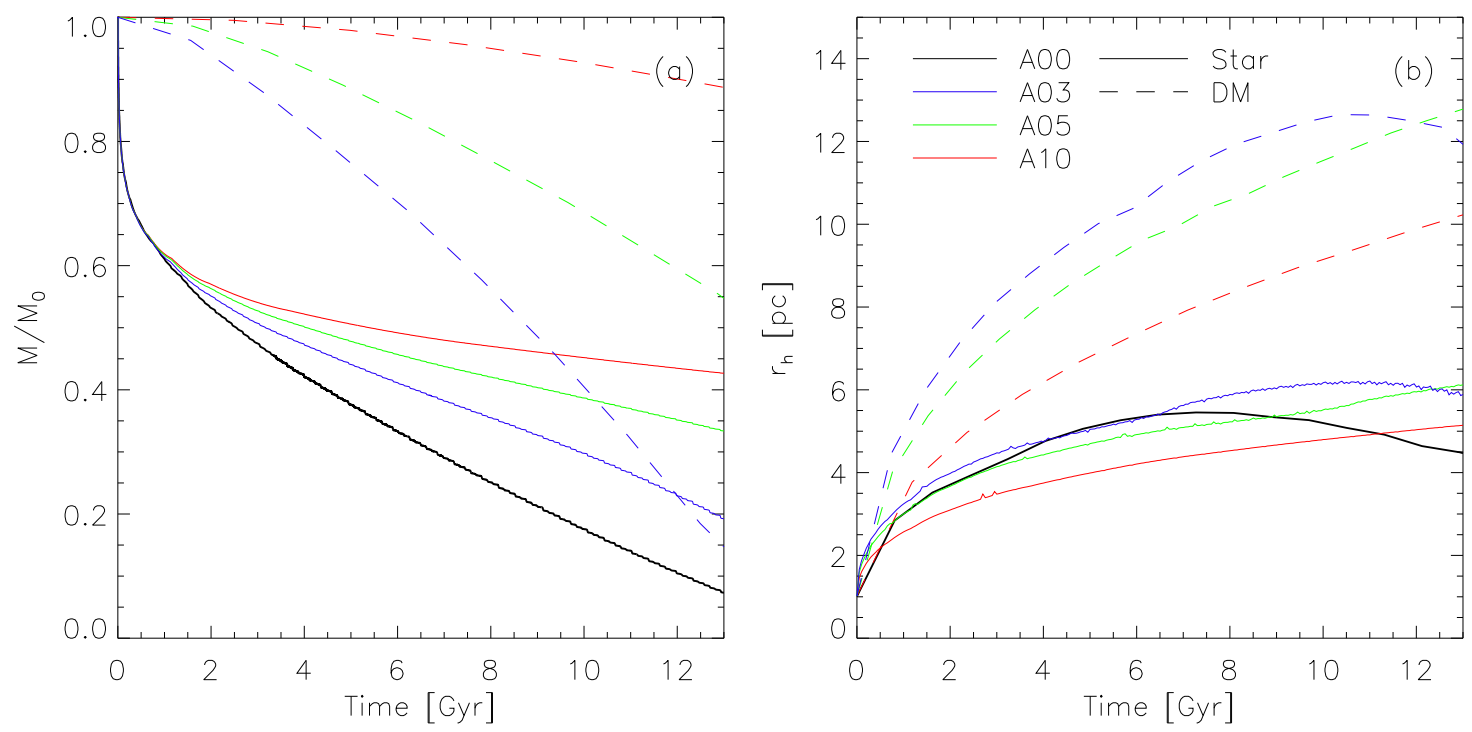

Fig. 1. - Evolution of $M$ (a) and $r_{\mathrm{h}}$ (b) for models A00 (black), A03 (blue), A05 (green), and A10 (red). Solid lines denote stars and dashed lines denote dark matter. See the electronic edition of the Journal for a color version of this figure.

Stars and DM particles whose apocenters are beyond the tidal radius of the cluster were removed from our FP calculations. Stars and DM particles cannot immediately escape the cluster, as it requires some amount of time for them to travel far enough from the cluster's Roche lobe (Lee \& Ostriker 1987), even if they were to satisfy the escape criterion. For the escapers, we thus followed the treatment of Takahashi \& Portegies Zwart (1998), who considered the escape timescale into their FP model using a formalism of Lee \& Ostriker (1987).*

For the initial stellar mass function of the cluster, we adopted the model developed by Kroupa (2001) with a mass range of $0.08-15 \mathrm{M}_{\odot}$, which was realized by 30 discrete mass components. Each mass component of stars followed the stellar evolution recipe described by Schaller et al. (1992). The stellar density and velocity dispersion distributions initially followed the isotropic King model (King 1966), with no initial mass segregation. For the DM component, we fixed $m_{\mathrm{DM}}$ to be $0.0001 \mathrm{M}_{\odot}$, which is small enough compared to $m_{\text {star }}$. The initial density and velocity dispersion distributions of the DM also followed the King model. For simplicity, we set $W_{0}$ of the DM component to be same as that of the stellar component.

* Originally, we removed DM particles instantaneously in our FP calculations on the ground that the relaxation timescale of the $\mathrm{DM}$ is much longer than the orbital timescale on which the escapes take place. However, Hyung Mok Lee, the referee of the present paper, made us realize that such instantaneous removal is appropriate only when the DM is the only component in the cluster. We have carried out all FP calculations in the present study again with the same removal prescription for both components (the new results are not considerably different from the original results because the relaxation timescale of the stars are much longer than the orbital timescale as well). We appreciate the referee for correcting this.

\section{EVOLUTION OF GCS WITH DM}

We first analyzed the characteristics of the dynamical evolution of GCs when they initially contain a non-negligible amount of DM. Five models with different initial conditions were used for the analysis in this section. The model parameters are given in Table 1. Model A had a relatively short $t_{\mathrm{rh}, \mathrm{star}}, 0.1 \mathrm{Gyr}$, and the $t_{\mathrm{rh} \text {,star }}$ of Model B was 50 times longer, 5 Gyr. The numbers in the model names denote the mass ratio between the DM and the stars $\left(M_{\mathrm{DM}} / M_{\text {star }}=0.0,0.3\right.$, 0.5, and 1.0). All five models had the same initial King concentration parameter $\left(W_{0}\right)$ of 4 .

The evolution of the mass $M$ and $r_{\mathrm{h}}$ of models A00, A03, A05, and A10, which have the same initial conditions for the stellar component, are shown in Fig. 1. $M_{\text {star }}$ steeply decreased in the early evolutionary stage, in which the stellar evolution (mass losses and supernova explosions) of the massive stars drives the evolution of the whole GC ( $\lesssim 1 \mathrm{Gyr})$; however, $M_{\mathrm{DM}}$ had only a mild mass loss during the same period. Once the mass segregation between the DM and stellar components becomes more important than the stellar evolution, the mass loss rate of the DM component surpasses that of the stellar component (see Fig. 1b); the outer part of the cluster is dominated by the DM component, making the DM component more vulnerable to loss over the Roche lobe of the cluster.

For the same initial $M_{\text {star }}$, a cluster with a larger initial $M_{\mathrm{DM}}$ loses both stellar and DM components less rapidly. This is because a deeper gravitational potential caused by the larger $M_{\mathrm{DM}}$ leads to a larger stellar velocity dispersion, which then results in a longer relaxation timescale for the stellar population (the local relaxation timescale is proportional to $v_{\mathrm{rms}}^{3} \rho^{-1} m^{-1}$; 

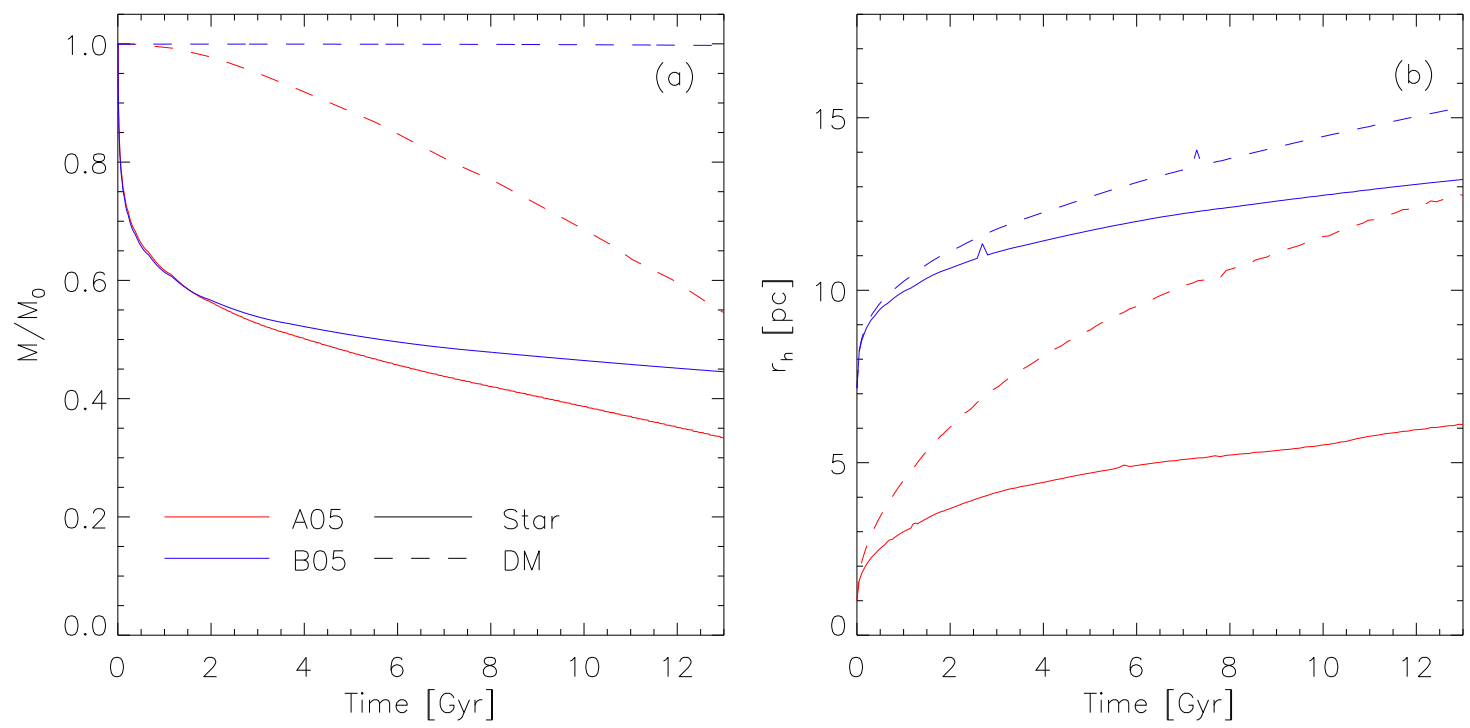

Fig. 2.- Evolution of $M(\mathrm{a})$ and $r_{\mathrm{h}}(\mathrm{b})$ for models A05 (red) and B05 (blue). Solid lines denote stars and dashed lines denote dark matter. See the electronic edition of the Journal for a color version of this figure.

Spitzer 1987). A longer relaxation timescale, in turn, results in a slower dynamical evolution of the stellar population, as well as a slower mass segregation between the stellar and DM components. It is important to note that the timescale of the two-body relaxation between DM particles is extremely long, because of the $m^{-1}$ dependence of the local relaxation timescale (this is why $D_{E_{\mathrm{DM}}}$ and $D_{R_{\mathrm{DM}}}$ were assumed to be zero in Section 2). Thus, the evolution of the DM component is solely driven by its interaction with the stellar component.

Comparisons of the $M$ and $r_{\mathrm{h}}$ evolutions between the models with two different $t_{\text {rh,star values (Models }}$ A05 and B05) are presented in Fig. 2. While Model A05, which had a 50 times shorter $t_{\text {rh,star than Model }}$ B05, lost a significant amount of DM within the Hubble time, the DM loss in model B05 was negligible. This comparison drastically shows the role of the mass segregation between the stellar and DM components in determining the fate of the DM component.

\section{INITIAL DM CONTENT OF NGC 6397}

NGC 6397 is a typical Galactic GC, which currently has a short $t_{\text {dep }}\left(\sim 1\right.$ Gyr) and an $R_{\mathrm{G}}$ of $\sim 6 \mathrm{kpc}$ (Harris 1996, 2010 edition). Unlike NGC 2419, which has a much longer $t_{\text {dep }}(\sim 100 \mathrm{Gyr})$ and a much larger $R_{G}$ ( $90 \mathrm{kpc}$ ), the DM component of NGC 6397 would have suffered a significant amount of dynamical segregation and mass loss over the tidal radius. Thus, the amount of DM remaining within NGC 6397 at this time is expected to be relatively small compared to the initial amount, even if it initially had a significant amount of DM. In this section, we search for the initial conditions that best-fit the current NGC 6397 by calculating the dynamical evolution of GCs using the FP models.

\subsection{Observational Data of NGC 6397}

NGC 6397 is an 'old halo' cluster, which is believed to have been created when the Milky Way protogalaxy collapsed (Zinn 1993; Mackey \& van den Bergh 2005), and is classified as an core-collapsed cluster (Trager et al. 1995). Its current total mass is estimated to be $\sim 6.2 \times 10^{4} \mathrm{M}_{\odot}($ Mandushev et al. 1991), and its age to be $\sim 11.5$ Gyr (Hansen et al. 2007). The absolute magnitude $M_{\mathrm{V}}$ of the cluster is -6.63 , and the projected half-light radius $R_{\mathrm{h}}$ is $2.33^{\prime}$, which corresponds to $2.04 \mathrm{pc}$ with an estimated distance from the Sun of $2.3 \mathrm{kpc}$ (Harris 1996, 2010 ed.).

Clusters experience different strengths of disk/bulge shocks and tidal fields depending on their orbital trajectories. We traced back the trajectory of NGC 6397 in the Galactic potential for the whole lifetime of the cluster from its current position. For the current 3dimensional velocity, we used the kinematic data of NGC 6397 by Dinescu et al. (1999), and we adopted the Galactic potential model by Johnson, Spergel, \& Hernquist (1995). For simplicity, we assumed that the orbit of NGC 6397 does not decay due to the dynamical friction, since the decaying timescale of NGC 6397 to the Galactic center is much larger than the Hubble time. The apocenter and pericenter distances of NGC 6397 are $8.0 \mathrm{kpc}$ and $2.8 \mathrm{kpc}$, respectively (orbital eccentricity of $\sim 0.47)$. To consider the time-varying Galactic tides due to the eccentric orbit, we continuously updated the size of the Roche lobe of the cluster, which is determined by the current $M$ and $R_{G}$ for each FP time-step (Shin, Kim, \& Takahashi 2008).

For the comparisons between the observed NGC 
Table 2.

Best-fit initial conditions for NGC 6397 for a given $r_{\mathrm{h}, \mathrm{DM}} / r_{\mathrm{h}, \mathrm{star}}$

\begin{tabular}{|c|c|c|c|c|c|c|c|c|}
\hline \multirow[b]{2}{*}{ Model } & \multicolumn{3}{|c|}{ star } & \multicolumn{2}{|c|}{$\mathrm{DM}$} & \multirow[b]{2}{*}{$M / L^{\mathrm{a}}$} & \multirow[b]{2}{*}{$\chi^{2}$} & \multirow[b]{2}{*}{$\begin{array}{c}p \text { value } \\
(\%)\end{array}$} \\
\hline & $\begin{array}{l}M_{\text {star }} \\
\left(\mathrm{M}_{\odot}\right)\end{array}$ & $\begin{array}{c}r_{\mathrm{h}, \mathrm{star}} \\
(\mathrm{pc})\end{array}$ & $\overline{W_{0}}$ & $M_{\mathrm{DM}} / M_{\text {star }}$ & $r_{\mathrm{h}, \mathrm{DM}} / r_{\mathrm{h}, \mathrm{star}}$ & & & \\
\hline $\mathrm{C}$ & $3.0 \times 10^{5}$ & 2.0 & 7.0 & 0.0 & - & 1.65 & 5.47 & 36.2 \\
\hline $\mathrm{D}$ & $2.5 \times 10^{5}$ & 1.3 & 4.9 & 0.3 & 1 & 3.98 & 7.12 & 21.2 \\
\hline $\mathrm{E}$ & $2.1 \times 10^{5}$ & 2.0 & 5.1 & 0.5 & 3 & 3.67 & 8.53 & 12.9 \\
\hline $\mathrm{F}$ & $2.8 \times 10^{5}$ & 1.6 & 5.3 & 0.3 & 5 & 2.93 & 6.28 & 28.0 \\
\hline
\end{tabular}

a Mass-to-light ratio.
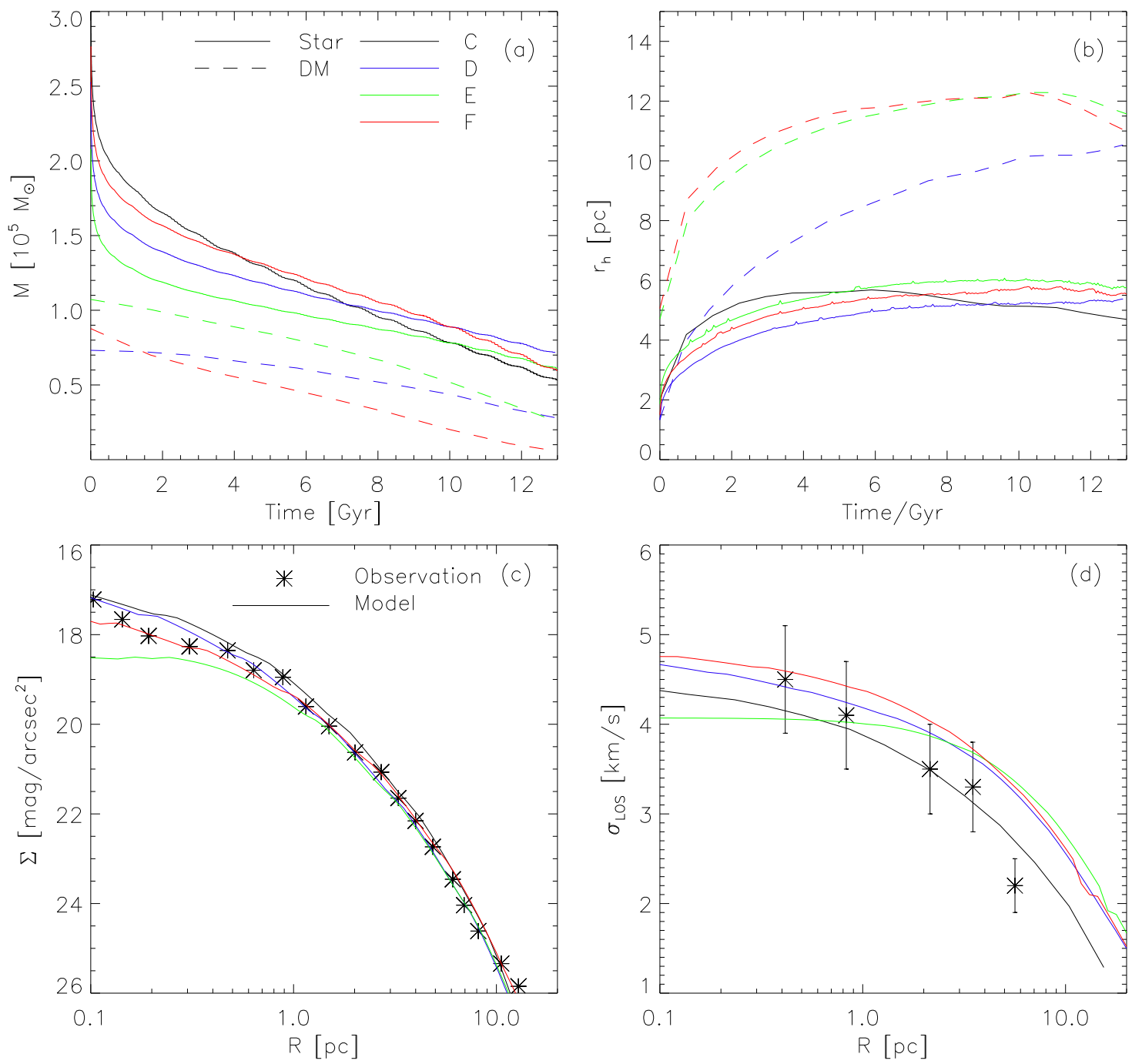

Fig. 3.- $M$ (a) \& $r_{\mathrm{h}}$ (b) evolution and $\Sigma$ (c) \& $\sigma_{\mathrm{LOS}}$ (d) profiles for Models C (black), D (blue), E (green), and F (red). Solid lines denote stars and dashed lines denote dark matter in panels a and b. Observed $\Sigma$ and $\sigma_{\text {LOS }}$ profiles of NGC 6397 are denoted with asterisks in panels c and d. See the electronic edition of the Journal for a color version of this figure. 
6397 and our FP results, we used the surface brightness profile $\Sigma(R)$ observed in the $\mathrm{V}$-band by Trager et al. (1995) and the line-of-sight velocity dispersion profile $\sigma_{\text {LOS }}(R)$ observed for giants and subgiants by Meylan \& Mayor (1991). Panels c and d of Figs. 3 and 4 show the adopted observations of NGC $6397, \Sigma(R)$ and $\sigma_{\mathrm{LOS}}(R)$.

We obtained the model $\Sigma(R)$ by applying the Abel integration to the three-dimensional mass density profile $\rho(r)$ of the FP results and then transforming the surface mass density to the V-band surface brightness using the stellar mass-luminosity relation of the YonseiYale model (Dermarque et al. 2004) and a metallicity of $[\mathrm{Fe} / \mathrm{H}]=-1.95$ (Harris 1996). Note that $R$ denotes the two-dimensional projected radius, and $r$ denotes the three-dimensional radius.

We obtained $\sigma_{\text {LOS }}(R)$ from the 3-dimensional tangential and radial velocity profiles, $\sigma_{\mathrm{t}}(r)$ and $\sigma_{\mathrm{r}}(r)$, for the mass components that correspond to the giants and subgiants using the following relations (Genzel et al. 2000):

$$
\begin{aligned}
\Sigma(R) \sigma_{\mathrm{LOS}}^{2}(R)= & 2 \int_{R}^{\infty}\left[\sigma_{\mathrm{r}}^{2}(r)(1-(R / r))^{2}\right. \\
& \left.+\sigma_{\mathrm{t}}^{2}(r)(R / r)^{2}\right] \times \frac{\Sigma(r) r \mathrm{dr}}{\left(r^{2}-R^{2}\right)^{1 / 2}} .
\end{aligned}
$$

\subsection{Best-fit Initial Conditions of NGC 6397}

To find the best-fit initial conditions that evolved into the current NGC 6397, we performed more than one hundred FP calculations using different combinations of the initial parameters: $M_{\mathrm{star}}, r_{\mathrm{h}, \mathrm{star}}, W_{0}$, $M_{\mathrm{DM}} / M_{\mathrm{star}}$, and $r_{\mathrm{t}, \mathrm{DM}} / r_{\mathrm{t} \text {,star }}$, where $r_{\mathrm{t}}$ is the tidal radius of the King profile. For $r_{\mathrm{t}, \mathrm{DM}} / r_{\mathrm{t}, \mathrm{star}}$, we tried only four different values, 0 (no DM), 1, 3, and 5 .

For each $r_{\mathrm{t}, \mathrm{DM}} / r_{\mathrm{t}, \mathrm{star}}$ value, we found an initial condition that best fit both the current, observed $\Sigma(R)$ and $\sigma_{\mathrm{LOS}}(R)$ of NGC 6397 . When finding the best-fit initial conditions, we employed a simple trial-and-error method: we kept changing one or two parameters at each trial until we find the set of parameters that yield the smallest $\chi^{2}$ value. These best-fit initial conditions obtained in this way are listed in Table 2 .

Models C, D, E, and F were the best-fit models for $r_{\mathrm{t}, \mathrm{DM}} / r_{\mathrm{t}, \mathrm{star}}=0,1,3$, and 5 , respectively. These four best-fit models gave acceptably high $p$ values (see Table 2). ${ }^{\dagger}$ We found that it is relatively easy to fit the observed surface brightness profile (see Fig. 3c). However, as seen in Fig. 3d, models with a DM component have $\sim 0.5$ dex higher $\sigma_{\text {LOS }}$ values in the outskirts of the cluster than models without a DM component, and all of the models with a DM component showed a nonnegligible deviation from the outermost observational

\footnotetext{
${ }^{\dagger}$ The $p$ value is the probability of having a $\chi^{2}$ that is larger than the value obtained from our $\chi^{2}$ test between the model and the observation, whose degree of freedom is 5 .
}

data point of $\sigma_{\text {LOS }}$. This is because the presence of DM causes a deeper gravitational potential, and thus higher stellar velocity dispersions.

We were not able to determine a set of initial conditions that fit the observations with $M_{\mathrm{DM}} / M_{\text {star }} \gtrsim 1$, and the maximum value of $M_{\mathrm{DM}} / M_{\text {star }}$ among our best-fit models with a DM component (models D, E, and F) was 0.5 . This $M_{\mathrm{DM}} / M_{\text {star }}$ value corresponds to a mass-to-light ratio $(M / L)$ of 3.7 , using the stellar population left in the cluster at 11.5 Gyr. This $M / L$ value is similar to those of ultra compact dwarf (UCD) galaxies and is much smaller than those of ultra faint dwarf (UFD) galaxies (GCs and UCDs typically have $M / L \sim 2$ and $\sim 4$, respectively, and UFDs mostly have $M / L>100$; Mieske et al. 2008, Simon \& Geha 2007). UCDs and UFDs are the closest sub-galactic systems to the GCs in terms of mass; however, GCs and UCDs are thought to have very different formation scenarios from UFDs, due to the current absence of a significant amount of DM in GCs. Our results show that NGC 6397 was either formed with only a small amount of DM content, if any, or NGC 6397 must have lost a considerable amount of DM in the very early phase of its lifetime, if it were formed with as much DM as UFDs or any other type of galaxy.

Both NGC 6397 and NGC 2419 appear to have had only a small amount of DM from the early phase, but this does not necessarily imply that the two clusters had similar origins. While NGC 6397 is a typical 'old halo' GC of the Milky Way, NGC 2419 has a very different stellar population from those of the Milky Way GCs, thus is thought to be the core of an accreted dwarf galaxy (Cohen \& Kirby 2012; Lee, Y.-W., et al. 2013, in preparation). It is highly probable that the progenitor of NGC 2419 had lost the majority of not only the stellar halo, but also its DM component, during the accretion process to the Milky Way.

As mentioned above, more than one hundred FP calculations were performed in order to determine the best-fit initial models for four different values of $r_{\mathrm{t}, \mathrm{DM}} / r_{\mathrm{t}, \mathrm{star}}$. To show the dependence of the initial conditions on the present-day $\Sigma$ and $\sigma_{\text {LOS }}$ profiles, we compared FP calculations of the six models whose initial conditions were similar to Model D. Each of the Models, Da through Df, had one variation on the initial conditions of Model D (see Table 3): Models Da and Db had different $M_{\text {star }}$ values, Models Dc and Dd had different $r_{\mathrm{h}}$ values, and Models De and Df had different $M_{\mathrm{DM}} / M_{\text {star }}$ values than Model D. ${ }^{\ddagger}$ Panels c and d of Fig. 4 show the present-day $\Sigma$ and $\sigma_{\text {LOS }}$ profiles of Models D and Da through Df. Models Da through Df gave $\Sigma$ and $\sigma_{\text {LOS }}$ profiles that were systematically below or above Model D in part or all of the radial range. One exception is the $\Sigma$ profile of Model Db,

\footnotetext{
$\ddagger$ Here we do not present the models that initially have different $W_{0}$ values from Model $\mathrm{D}$, because those models result in $\Sigma$ and $\sigma_{\mathrm{LOS}}$ profiles very similar to those of Model D. $\Sigma$ and $\sigma_{\mathrm{LOS}}$ profiles are rather insensitive to initial $W_{0}$.
} 
Table 3.

Initial conditions for Models Da-Df with variations from Model D.

\begin{tabular}{|c|c|c|c|c|c|c|c|c|}
\hline \multirow[b]{2}{*}{ Model } & \multicolumn{3}{|c|}{ star } & \multicolumn{2}{|c|}{$\mathrm{DM}$} & \multirow[b]{2}{*}{$M / L^{\mathrm{a}}$} & \multirow[b]{2}{*}{$\chi^{2}$} & \multirow[b]{2}{*}{$\begin{array}{c}p \text { value } \\
(\%)\end{array}$} \\
\hline & $\begin{array}{l}M_{\text {star }} \\
\left(\mathrm{M}_{\odot}\right)\end{array}$ & $\begin{array}{c}r_{\mathrm{h}, \mathrm{star}} \\
(\mathrm{pc})\end{array}$ & $\overline{W_{0}}$ & $M_{\mathrm{DM}} / M_{\text {star }}$ & $r_{\mathrm{h}, \mathrm{DM}} / r_{\mathrm{h}, \mathrm{star}}$ & & & \\
\hline $\mathrm{D}$ & $2.5 \times 10^{5}$ & 1.3 & 4.9 & 0.3 & 1 & 3.98 & 7.12 & 21.2 \\
\hline $\mathrm{Da}$ & $2.0 \times 10^{5}$ & 1.3 & 4.9 & 0.3 & 1 & 3.10 & 10.72 & 5.72 \\
\hline $\mathrm{Db}$ & $3.0 \times 10^{5}$ & 1.3 & 4.9 & 0.3 & 1 & 4.35 & 20.25 & 0.11 \\
\hline Dc & $2.5 \times 10^{5}$ & 0.3 & 4.9 & 0.3 & 1 & 2.52 & 18.71 & 0.22 \\
\hline $\mathrm{Dd}$ & $2.5 \times 10^{5}$ & 2.3 & 4.9 & 0.3 & 1 & 3.85 & 23.74 & 0.02 \\
\hline De & $2.5 \times 10^{5}$ & 1.3 & 4.9 & 0.1 & 1 & 2.18 & 14.11 & 1.49 \\
\hline $\mathrm{Df}$ & $2.5 \times 10^{5}$ & 1.3 & 4.9 & 0.5 & 1 & 5.42 & 12.33 & 3.05 \\
\hline
\end{tabular}

${ }^{a}$ Mass-to-light ratio.
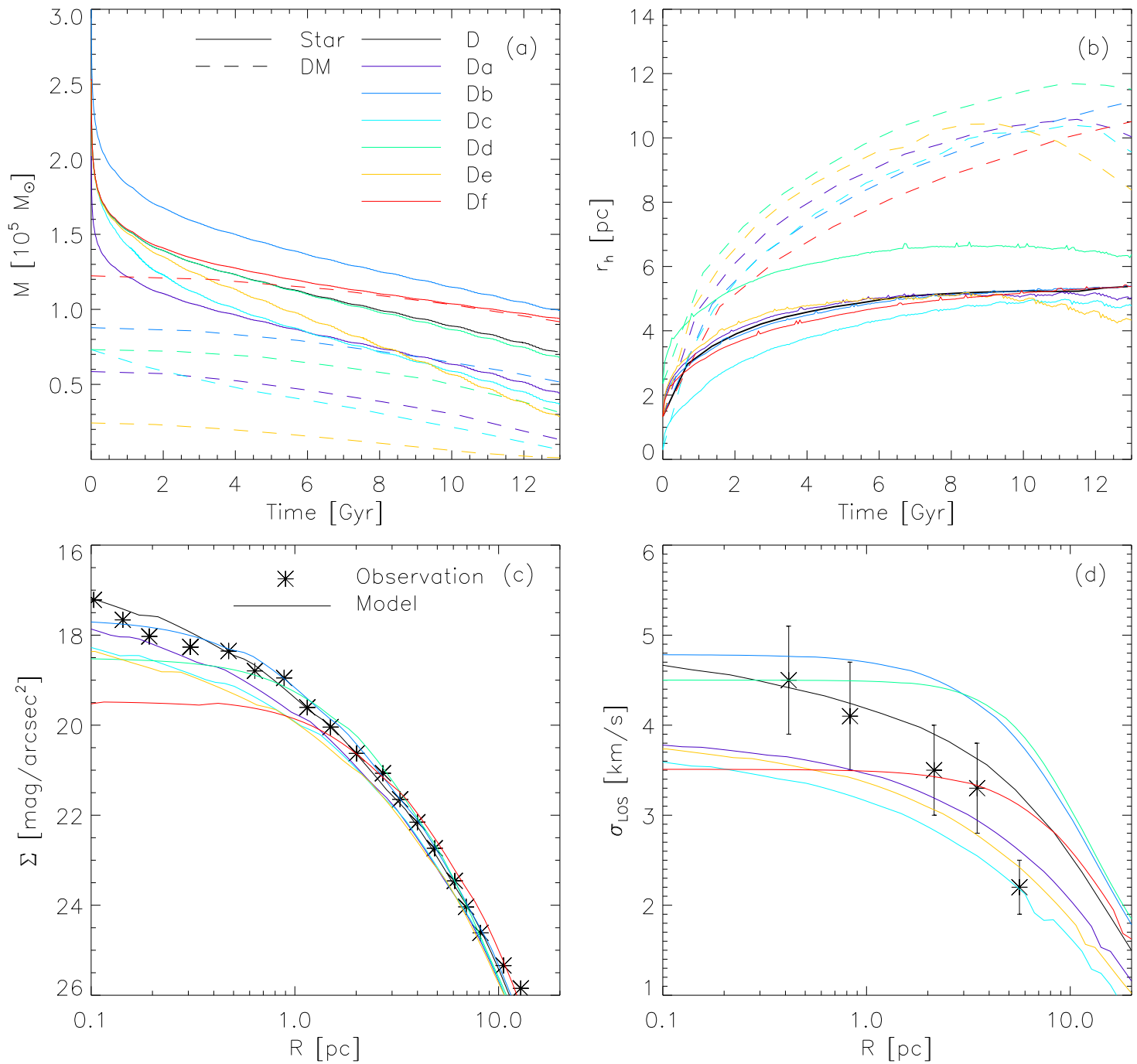

Fig. 4. - Same as Fig. 3, but for Models D and Da-Df. See the electronic edition of the Journal for a color version of this figure. 
which was quite similar to that of Model D; however its $\sigma_{\text {LOS }}$ profile was still very different from that of Model D. Quantitatively, the $p$ values of Models Da through Df were all unacceptably small, except for Model Da, and considerably smaller than that of Model D. These qualitative and quantitative comparisons give an idea of the procedure that we followed to find our best-fit models.

Note that we did not try $r_{\mathrm{t}, \mathrm{DM}} / r_{\mathrm{t}, \mathrm{star}}>5$ cases for NGC 6397, because the DM halo would overflow the tidal radius when $r_{\mathrm{t}, \mathrm{DM}} / r_{\mathrm{t} \text {,star }}>5$ and the Galactic native GCs, which were formed in the collapsing protogalaxy, are not likely to overfill the tidal radius initially.

\section{SUMMARY}

Using the most advanced FP models, we studied the dynamical evolution of GCs with a DM component. Since the DM potential increases the velocity dispersion of stars, the existence of DM delays the dynamical evolution of stars in the GC compared to that of a cluster without a DM component. Through the mass segregation between DM and stars, the DM component gradually migrates to the outer part of the GC, and the mass loss rate of the DM component becomes larger than that of the stellar component. Since the depletion of the DM in the inner part of the GC is more prominent in GCs with a shorter $t_{\mathrm{rh} \text {,star, one can }}$ not directly infer the initial amount of the DM content of the short $t_{\mathrm{rh} \text {,star }}$ GCs without numerically modelling their dynamical evolutions.

With FP calculations, we traced the dynamical evolution of NGC 6397, a typical Galactic GC with a short $t_{\mathrm{rh}, \text { star }}(<0.3 \mathrm{Gyr})$ and $R_{\mathrm{G}}$ of $\sim 6 \mathrm{kpc}$. We found that the best-fit initial $M_{\mathrm{DM}} / M_{\text {star }}$ value for NGC 6397 is not more than 1 for $r_{\mathrm{t}, \mathrm{DM}} / r_{\mathrm{t}, \mathrm{star}}<5$. Moreover, the model without an initial DM content, Model C, had the highest p-value among the best-fit models. Therefore, we conclude that NGC 6397 did not initially contain a significant amount of DM, if any, and our conclusion is similar to the results for NGC 2419 by Baumgardt and Mieske (2008), and Conroy, Loeb, and Spergel(2011).

The initial $M_{\mathrm{DM}}$ for NGC 6397 could be larger than $\sim 0.3 M_{\text {star }}$, if most of the initial DM was stripped away from the GC during the first several orbits, when NGC 6397 was being accreted into the Galaxy. However, NGC 6397 is categorized as an 'old halo' cluster, and is believed to have formed inside a collapsing protogalaxy (Zinn 1993; Mackey \& van den Bergh 2005); therefore, it is more likely that NGC 6397 has not experienced any sudden stripping of DM. Therefore, the actual initial DM content of NGC 6397 was probably not much larger than what we found in this study.

\section{ACKNOWLEDGMENTS}

We thank Koji Takahashi for his help with his FP models. This work was supported by Mid-career Re- search Program (No. 2011-0016898) through the National Research Foundation (NRF) grant funded by the Ministry of Education, Science and Technology (MEST) of Korea. JS deeply appreciates Hansung Benjamin Gim for his help in selecting our target GC.

\section{REFERENCES}

Ashman, K. M., \& Zepf, S. E. 1992, The Formation of Globular Clusters in Merging and Interacting Galaxies, ApJ, 384, 50

Baumgardt, H., \& Mieske, S. 2008, High Mass-to-Light Ratios of Ultra-Compact Dwarf Galaxies - Evidence for Dark Matter?, MNRAS, 391, 942

Baumgardt, H., Côté, P., Hilker, M., Rejkuba, M., Mieske, S., Djorgovski, S. G., \& Stetson, P. 2009, The Velocity Dispersion and Mass-to-Light Ratio of the Remote Halo Globular Cluster NGC 2419, MNRAS, 396, 2051

Beasly, M. A., Kawata, D., Pearce, F. R., Forbes, D. A., \& Gibson, B. K. 2003, The Metallicity of Pregalactic Globular Clusters: The Observational Consequences of the First Stars, ApJ, 596, L187

Bekki, K., Yahagi, H., Nagashima, M., \& Forbes, D. A. 2007, Formation of the Galactic Globular Clusters with He-Rich Stars in Low-Mass Haloes Virialized at High Redshift, MNRAS, 382, L87

Boley, A. C., Lake, G., Read, J., \& Teyssier, R. 2009, Globular Cluster Formation Within a Cosmological Context, ApJ, 706, L192

Bromm, V., \& Clarke, C. J. 2002, The Formation of the First Globular Clusters in Dwarf Galaxies before the Epoch of Reionization, ApJ, 566, L1

Cen, R. 2001, Synchronized Formation of Subgalactic Systems at Cosmological Reionization: Origin of Halo Globular Clusters, ApJ, 560, 592

Cohen, J. G., \& Kirby, E. N. 2012, The Bizarre Chemical Inventory of NGC 2419, An Extreme Outer Halo Globular Cluster, ApJ, 760, 86

Conroy, C., Loeb, A., \& Spergel, D. N. 2011, Evidence against Dark Matter Halos Surrounding the Globular Clusters MGC1 and NGC 2419, ApJ, 741, 72

Demarque, P., Woo, J. H., Kim, Y. C., \& Yi, S. K. 2004, Y ${ }^{2}$ Isochrones with an Improved Core Overshoot Treatment, ApJS, 155, 667

Dinescu, D. I., Girard, T. M., \& van Altena, W. F. 1999, Space Velocities of Globular Clusters. III. Cluster Orbits and Halo Substructure, AJ, 117, 1792

Fall, S. M., \& Rees, M. J. 1985, A Theory for the Origin of Globular Clusters, ApJ, 298, 18

Genzel, R., Pichon, C., Eckart, A., Gerhard, O. E., \& Ott, T. 2000, Stellar Dynamics in the Galactic Centre: Proper Motions and Anisotropy, MNRAS, 317,348 
Griffen, B. F., Drinkwater, M. J., Thomas, P. A., \& Helly, J. C. 2010, Globular Cluster Formation within the Aquarius Simulation, MNRAS, 405, 375

Gunn, J. E. 1980, in Globular Clusters, ed. D. Hanes \& B. Madore, 301 (Cambridge: Cambridge University Press)

Hansen, B. M. S., Anderson, J., Brewer, J., Dotter, A., Fahlman, G. G., Hurley, J., Kalirai, J., King, I., Reitzel, D., Richer, H. B., Rich, R. M., Shara, M. M., \& Stetson, P. B. 2007, The White Dwarf Cooling Sequence of NGC 6397, ApJ, 671, 380

Harris, W. E. 1996, A Catalog of Parameters for Globular Clusters in the Milky Way, AJ, 112, 1487

Johnson, K. V., Spergel, D. N., \& Hernquist, L. 1995, The Disruption of the Sagittarius Dwarf Galaxy, ApJ, 451, 598

King, I. R. 1966, The Structure of Star Clusters. III. Some Simple Dynamical Models, AJ, 71, 64

Kroupa, P. 2001, On the Variation of the Initial Mass Function, MNRAS, 322, 231

Lee, H. M., \& Ostriker, J. 1987, The Evolution and Final Disintegration of Spherical Stellar Systems in a Steady Galactic Tidal Field, ApJ, 322, 123

Mashchenko, S., \& Sills, A. 2005, Globular Clusters with Dark Matter Halos. I. Initial Relaxation, ApJ, 619,243

Mackey, A. D., \& van den Bergh, S. 2005, The Properties of Galactic Globular Cluster Subsystems, MNRAS, 360, 631

Mandushev, G., Staneva, A., \& Spasova, N. 1991, Dynamical Masses for Galactic Globular Clusters, A\&A, 252, 94

Meylan, G., \& Mayor, M. 1991, Studies of Dynamical Properties of Globular Clusters. VI - The HighConcentration Cluster NGC 6397, A\&A, 250, 113

Mieske, S., Hilker, M., Jordán, A., Infante, L, KisslerPatig, M., Rejkuba, M., Richtler, T., Côté, P., Baumgardt, H., West, M. J., Ferrarese, L., \& Peng, E. W. 2008, The Nature of UCDs: Internal Dynamics from an Expanded Sample and Homogeneous Database, A\&A, 487, 921

Moore, B. 1996, Constraints on the Global Mass-toLight Ratios and on the Extent of Dark Matter Halos in Globular Clusters and Dwarf Spheroidals, ApJ, 461, L13

Peebles, P. J. E. 1984, Dark Matter and the Origin of Galaxies and Globular Star Clusters, ApJ, 277, 470

Peebles, P. J. E., \& Dicke, R. H. 1968, Origin of the Globular Star Clusters, ApJ, 154, 891

Saitoh, T. R., Koda, J., Okamoto, T., Wada, K., \& Habe, A. 2006, Tidal Disruption of Dark Matter Halos around Proto-Globular Clusters, ApJ, 640, 22
Schaller, G., Schaerer, D., Meynet, G., \& Maeder, A. 1992, New Grids of Stellar Models from 0.8 to 120 Solar Masses at $\mathrm{Z}=0.020$ and $\mathrm{Z}=0.001$, A\&AS, 96,269

Shin, J., \& Kim, S. S. 2007, Alternating Direction Implicit Method for Two-Dimensional FokkerPlanck Equation of Dense Spherical Stellar Systemsm, JKAS, 40, 91

Shin, J., Kim, S. S., \& Takahashi, K. 2008, Dynamical Evolution of the Mass Function and Radial Profile of the Galactic Globular Cluster System, MNRAS, 386, L67

Shin, J., Kim, S. S., Yoon, S. J., \& Kim, J. 2013, Initial Size Distribution of the Galactic Globular Cluster System, ApJ, 762, 135

Simon, J. D., \& Geha, M. 2007, The Kinematics of the Ultra-Faint Milky Way Satellites: Solving the Missing Satellite Problem, ApJ, 670, 313

Spitzer, L. Jr. 1987, Dynamical Evolution of Globular Clusters (Princeton: Princeton University Press)

Takahashi, K., \& Lee, H. M. 2000, Evolution of Multimass Globular Clusters in the Galactic Tidal Field with the Effects of Velocity Anisotropy, MNRAS, 316,671

Takahashi, K., \& Portegies Zwart, S. 1998, The Disruption of Globular Star Clusters in the Galaxy: A Comparative Analysis between Fokker-Planck and N-Body Models, ApJ, 503, L49

Takahashi, K., Sensui, T., Funato, Y., \& Makino, J. 2002, Collisional Evolution of Galaxy Clusters and the Growth of Common Halos, PASJ, 54, 5

Takahashi, K. 1997, Fokker-Planck Models of Star Clusters with Anisotropic Velocity Distributions III. Multi-Mass Clusters, PASJ, 49, 547

Trager, S. C., King, I. R., \& Djorgovski, S. 1995, Catalogue of Galactic Globular-Cluster SurfaceBrightness Profiles, AJ, 109, 218

Zinn, R. 1993, The Galactic Halo Cluster Systems: Evidence for Accretion, ASPC, 48, 39 